\title{
Corpus callosotomy via laser interstitial thermal therapy: a case series
}

\author{
Atilio E. Palma, MD, ${ }^{1}$ Robert T. Wicks, MD, ${ }^{1}$ Gautam Popli, MD, ${ }^{2}$ and Daniel E. Couture, MD ${ }^{1}$ \\ Departments of ${ }^{1}$ Neurological Surgery and ${ }^{2}$ Neurology, Wake Forest University School of Medicine, Winston-Salem, North \\ Carolina
}

\begin{abstract}
Corpus callosotomy has been used as a form of surgical palliation for patients suffering from medically refractory generalized seizures, including drop attacks. Callosotomy has traditionally been described as involving a craniotomy with microdissection. MR-guided laser interstitial thermal therapy (MRg-LITT) has recently been used as a minimally invasive method for performing surgical ablation of epileptogenic foci and corpus callosotomy. The authors present 3 cases in which MRg-LITT was used to perform a corpus callosotomy as part of a staged surgical procedure for a patient with multiple seizure types and in instances when further ablation of residual corpus callosum is necessary after a prior open surgical procedure. To the authors' knowledge, this is the first case series of corpus callosotomy performed using the MRg-LITT system with a 3.3-year average follow-up. Although MRg-LITT is not expected to replace the traditional corpus callosotomy in all cases, it is a safe, effective, and durable alternative to the traditional open corpus callosotomy, particularly in the setting of a prior craniotomy.

https://thejns.org/doi/abs/10.3171/2018.10.PEDS18368
\end{abstract}

KEYWORDS corpus callosotomy; laser interstitial thermal therapy; drop attacks; epilepsy

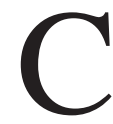
ORPUS callosotomy as a method of seizure containment was first described by Van Wagenen in the $1940 \mathrm{~s}^{1,13,20}$ In his series of patients with glioblastoma of the corpus callosum, in whom seizures were common, Van Wagenen noted that as the tumors progressed, destroying the corpus callosum, the frequency of seizures decreased. He also noted 3 other instances in which damage to the corpus callosum by a meningioma, ischemia, and hemorrhage led to a decrease in the frequency of seizures. He then set out to surgically divide the corpus callosum in 10 patients in order to minimize the spread of seizures from one hemisphere to the other. Bogen et al. further emphasized the benefits of corpus callosotomy in the 1960s, and corpus callosotomy continued to gain favor as a palliative procedure to reduce disabling generalized seizures. ${ }^{3,22}$ Although corpus callosotomy does not stop seizures per se, it does prevent the seizure from generalizing. Corpus callosotomy has been noted to be especially successful in treating drop attacks. .,10,23 $^{2}$

Corpus callosotomy has traditionally been performed using microsurgery. Minimally invasive procedures per- formed using endoscopy or stereotactic radiation have been described..$^{8,17}$ Conceived in the 1980s, MR-guided laser interstitial thermal therapy (MRg-LITT), a common treatment for ablation of small $(<3 \mathrm{~cm})$ tumors, has experienced increasing popularity for the treatment of focal epileptogenic lesions. ${ }^{21}$ The advent of MRI thermography during the 1990s made MRg-LITT a conventional and useful treatment modality for a variety of pathologies. During the procedure, a lesion is localized using neuroimaging and neuronavigation techniques, and a trajectory is planned. The laser probe is inserted stereotactically, aided by a frame-based or frameless stereotactic system. Initially used to treat inaccessible tumors, the use of MRgLITT has expanded over the years as experience with the technique has grown. Curry et al. first described the use of MRg-LITT for medically refractory epilepsy in 2012. ${ }^{6}$ Indications now include surgically inaccessible or difficult gliomas, metastases, radiation necrosis, spinal tumors, and epileptogenic lesions such as mesial temporal lobe epilepsy or focal cortical dysplasia., 2,4,7,14,15,19,24

MRg-LITT has the potential to offer a minimally inva-

ABBREVIATIONS CVEEG = continuous video electroencephalography; MRg-LITT = MR-guided laser interstitial thermal therapy; OR = operating room. SUBMITTED June 12, 2018. ACCEPTED October 9, 2018.

INCLUDE WHEN CITING Published online December 21, 2018; DOI: 10.3171/2018.10.PEDS18368. 
sive methodology for performing a successful corpus callosotomy, especially in cases in which a craniotomy or repeat craniotomy would be suboptimal. To our knowledge, only 2 other case series in which MRg-LITT had been used for corpus callosotomy exist. ${ }^{15,18}$

\section{Case Reports Case 1}

A 13-year-old boy who initially presented at the age of 8 years with 2 seizures, each of different semiology. His initial event consisted of a generalized seizure with a drop attack, during which he demonstrated generalized tonic-clonic activity for approximately 2 minutes. He had a subsequent seizure 2 weeks later with right head deviation, right-sided stiffening, and a typical postictal period of confusion. Results of routine electroencephalography (EEG) studies performed during his initial hospitalization were normal. An MRI study was performed, revealing multiple gray matter heterotopia lining the lateral ventricles. His seizures were initially managed with levetiracetam with temporary relief.

He then developed new seizure semiology, with tongue clicking and left facial twitching without loss of consciousness, several months after his initial seizure-free period. Carbamazepine, clobazam, and Depakote were added to his regimen, with little result. He was experiencing more than 10 drop attacks per week. He was evaluated in the epilepsy monitoring unit for medical optimization of seizure control. During his stay, 2 seizure types were observed: 1) drop-attack seizures associated with aphasia, and 2) episodes of right head deviation, drawing of the mouth, right-sided stiffening, and unresponsiveness. Magnetoencephalography demonstrated multifocal epileptiform activity, strongest from the parietal cortices. Continuous video EEG (cVEEG) showed abnormal runs of generalized spike and wave discharges with left hemispheric predominance. Several surgical options were proposed, including MRg-LITT ablation of heterotopia, MRg-LITT corpus callosotomy, and craniotomy for corpus callosotomy. The decision was made to stage MRgLITT ablation of the heterotopias, followed by MRg-LITT corpus callosotomy. Three lesions in the left hemisphere were selected for ablation due to their proximity to each other and localization of abnormal spikes on cVEEG during monitoring.

After this first stage of laser ablation, the patient's seizure frequency and severity were markedly reduced. His residual seizures were described as behavioral arrest and rare secondary generalization. He also had a reduction in drop events to 3-6 per week. He was taken to the operating room (OR) 6 months later for MRg-LITT ablation of the anterior two-thirds of the corpus callosum (Fig. 1). He did well postoperatively and was discharged home on postoperative day 2 .

His seizure frequency decreased to $2-3$ seizures per week with no drop attacks. Additionally, his affect and cognition improved. The seizures have further decreased with optimized antiepileptic drug dosing to 1-2 per week, without drop attacks. This effect has been maintained through 4 years of follow-up.
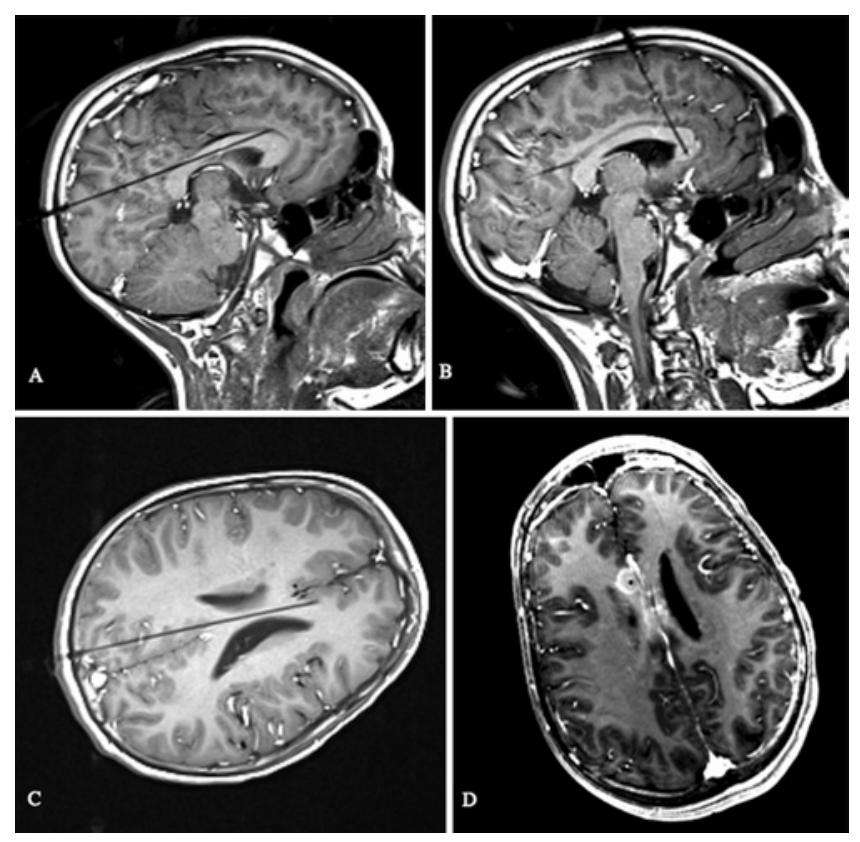

FIG. 1. MRI sequences of the brain. A: Sagittal T1-weighted image showing laser catheter trajectory on the body of the corpus callosum. B: Sagittal T1-weighted image showing laser catheter trajectory through the rostrum of the corpus callosum. C: Axial T1-weighted image showing laser catheter trajectory through the body of the corpus callosum. D: Axial T1-weighted image with addition of contrast showing part of the area of thermal ablation of the corpus callosum.

\section{Case 2}

A 23-year-old woman who had a past medical history of a stroke in the perinatal period that resulted in medically refractory epilepsy underwent vagal nerve stimulator placement at 14 years of age, followed by a right-sided hemispherectomy at 17 years of age, which gave her temporary improvement in her seizure control. She continued to experience between 40 and 60 seizures per month after surgery. Prior to hemispherectomy she would experience tonic seizures with drop attacks. After surgery she was able to catch herself, most of the time, before falling.

An MRI scan was performed as part of her ongoing seizure investigation, and showed she still had a small area of intact splenium of the corpus callosum. She underwent MRg-LITT ablation of the residual corpus callosum (Fig. 2 ) and was admitted to the ICU for close monitoring postoperatively. Her seizures improved and she ultimately saw complete resolution of drop attack, and continues to be free of drop attacks 3 years after MRg-LITT corpus callosotomy. This effect has been maintained through 3 years and 3 months of follow-up.

\section{Case 3}

A 13-month-old boy who had a past medical history of premature birth, hemimegalencephaly, and intractable seizures. Due to intractable epilepsy and disease severity he underwent left-sided hemispherectomy at 5 months of age. Postoperatively his seizure frequency had decreased significantly. He did well initially, but 2 months after surgery he began having 3-4 seizures per day, described as 

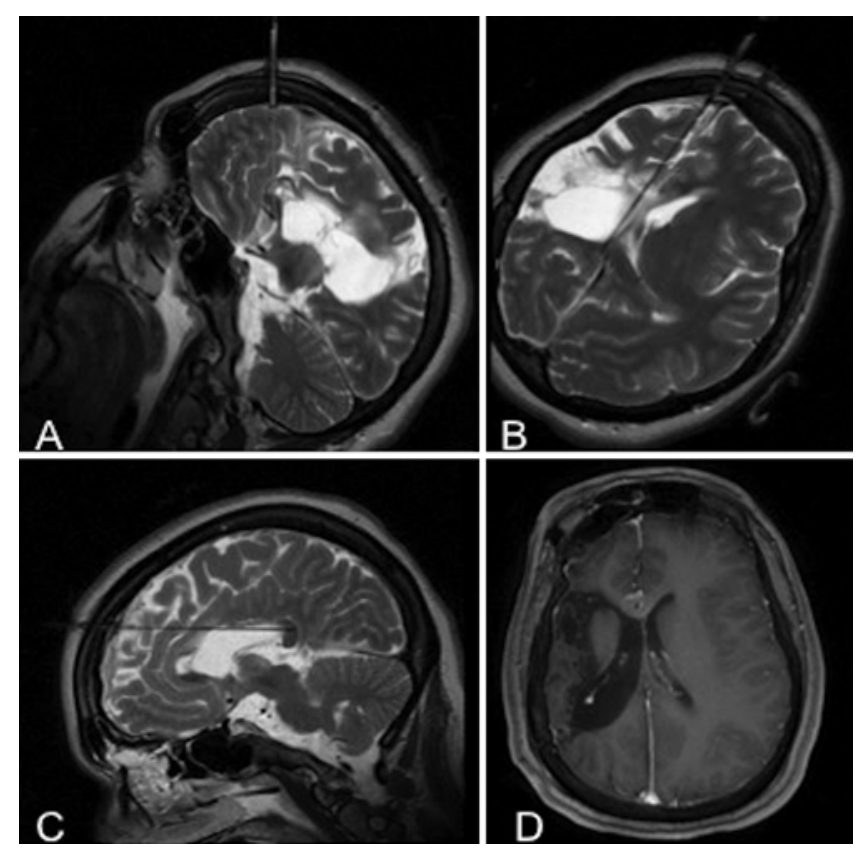

FIG. 2. MRI sequences of the brain. A: Oblique T2-weighted view of the laser catheter trajectory through the genu of the corpus callosum. B: Axial T2-weighted image showing the laser catheter trajectory through the body of the corpus callosum. C: Sagittal T2-weighted image showing the laser catheter trajectory through the body of the corpus callosum and ending in the splenium. D: Axial T1-weighted image obtained after addition of contrast showing a portion of the area of thermal ablation in the corpus callosum.

episodes of behavioral arrest that lasted a few seconds. These were accompanied by eye deviation to the right and dysconjugate eye movements. There was no postictal phase or cyanosis. He then developed new spells, with eye deviation to the left, his arms raised and crossed over his head, and his legs would cross and rise off the bed. He was placed on long-term EEG monitoring, which showed evidence of seizure activity within the left hemisphere spreading to the right. This was thought to be secondary to incomplete disconnection of tracts and pathways within the region of the corpus callosum. An MRI sequence of the brain with addition of contrast confirmed residual splenium of the corpus callosum. It was believed that his calvaria was thick enough $(4.7 \mathrm{~mm})$ to give adequate purchase for the LITT guidance bolt. The minimal blood loss associated with MRg-LITT made this procedure particularly attractive.

He was taken to the OR for MRg-LITT ablation of the residual corpus callosum (Fig. 3) and was discharged home on postoperative day 1 . He had an increase in seizure frequency initially, but ultimately reached near-complete resolution of his convulsive seizures. He was weaned off several antiepileptic medications over a 2-year period. He was also noted to have improved developmental progress after the corpus callosotomy, and has been free of drop attacks during a follow-up period of 2 years and 9 months.

\section{Operative Technique}

The patients in cases 1 and 2 were taken to the OR after placement of a Cosman-Roberts-Wells frame and after obtaining a CT scan of the head that would be fused with a preoperative MRI scan of the brain. A trajectory was planned that would begin at the skin and project through the intended target area. The patient in case 3 had a CT of the head performed prior to surgery, and the BrainLab Varioguide system was used to plan a trajectory that would project through the intended target area. The Visualase MRI-guided laser ablation system (Medtronic, Inc.) was used in each case. We performed the catheter placement in a similar fashion to that of Karsy et al., as recently published. ${ }^{12} \mathrm{~A}$ stab incision was made in the scalp and the laser probe was introduced stereotactically and secured by a guidance bolt used to direct the laser probe. In cases 1 and 2 an additional incision was made for a second laser trajectory in order to allow ablation of the intended portions of the corpus callosum. The patients were transported to the MRI unit where an MR image was obtained to ensure accurate laser placement. Lesioning was performed under MRI thermography to monitor the temperature changes
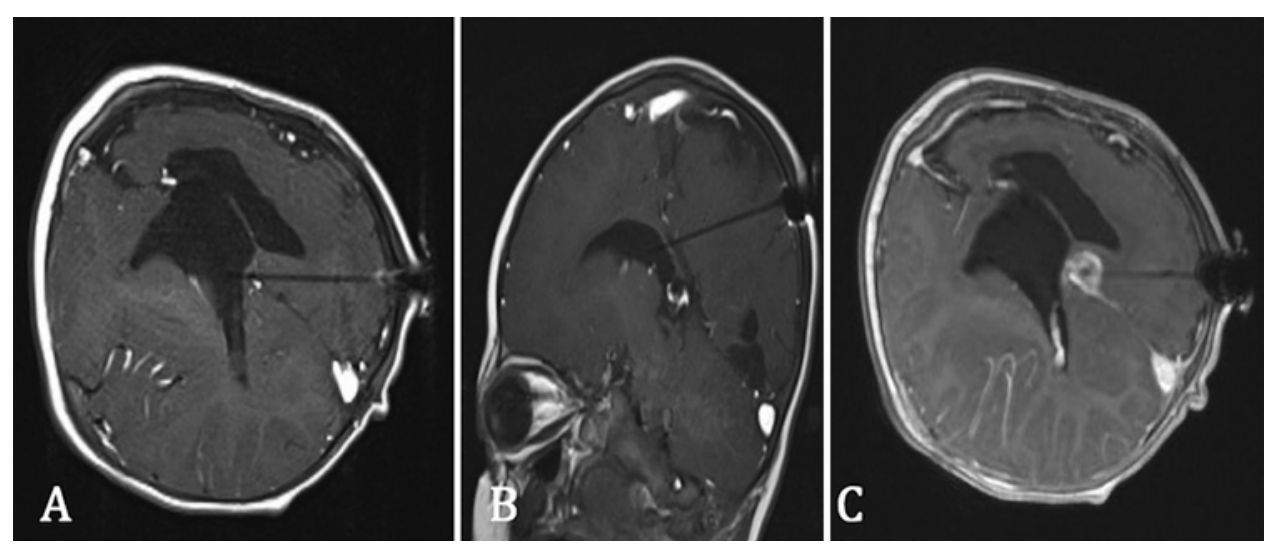

FIG. 3. MRI sequences of the brain. A: Axial T1-weighted image obtained after addition of contrast showing the laser catheter trajectory through the splenium of the corpus callosum. B: Sagittal T1-weighted image obtained after addition of contrast showing the laser catheter trajectory through the splenium of the corpus callosum. C: Axial T1-weighted image obtained after addition of contrast showing part of the area of thermal ablation within the region of the splenium of the corpus callosum. 
and damage in real time. After lesions were complete, an additional contrasted MRI sequence was obtained to visualize the lesions created, and the laser catheters were removed.

\section{Discussion}

The risks associated with craniotomy for corpus callosotomy have led some to attempt corpus callosotomy via less invasive means. Eder et al. reported on their case series of 3 patients who underwent stereotactic radiosurgery for corpus callosotomy. ${ }^{8}$ Two of the patients in their series had previously undergone functional hemispherectomy for cortical dysplasia, with radiosurgery used to complete a total callosotomy due to continued bihemispheric spread of ictal activity. The third child underwent radiosurgical callosotomy after right temporal resection for LennoxGastaut syndrome. They were able to achieve $100 \%$ reduction in secondarily generalized seizures and 20\%-70\% reduction of partial seizures in the children with hemispheric cortical dysplasia, but treatment failure occurred in the child with Lennox-Gastaut syndrome. Sood et al. reported on the use of the endoscope to perform a corpus callosotomy in 4 patients and hemispherotomy in 2 patients. ${ }^{17}$ This methodology, however, did require a 2- to $3-\mathrm{cm}$ craniotomy positioned laterally to the superior sagittal sinus.

A proof-of-concept study into the possible use of MRg-LITT to perform an anterior and complete corpus callostomy was recently proposed, ${ }^{16}$ and a recent report of successful laser ablation of the splenium of the corpus callosum for intractable epilepsy was published by Ho et al. ${ }^{11}$ As described by Esquenazi et al., MRg-LITT does not distort the gross anatomy or make subsequent resections more difficult, ${ }^{9}$ and it allows for surgical management, without increasing complexity, should laser ablation fail.

Two of the patients in our series had undergone a previous craniotomy for hemispherectomy. Their reluctance to undergo a second craniotomy to allow us to perform a corpus callosotomy made MRg-LITT corpus callosotomy a particularly good surgical option. MRg-LITT is a rapidly emerging technology for successful ablation of deep epileptogenic foci and, in our view, it is an effective and durable surgical technique for minimally invasive corpus callosotomy. To our knowledge, this is the first case series of corpus callosotomy performed using the MRg-LITT system with a 3.3-year average follow-up. It is also the first reported series of the use of MRg-LITT to complete disconnections in patients who have undergone a prior craniotomy. In our view, MRg-LITT is a safe, effective, and durable alternative to the traditional open corpus callosotomy.

Although safety and efficacy are the focus of this case series, the acute-care costs (both inpatient care and aftercare) as well as comfort and convenience to the patient must be considered in the use of new technology. Although the costs will vary between institutions, one must consider the cost of the technology (laser fibers), the OR time, the imaging studies, and the postoperative care. Although a statistical analysis of this small series is not possible, at our institution the OR time is actually increased in MRg-
LITT cases (average 5 hours 10 minutes for MRg-LITT vs 2 hours 58 minutes for traditional corpus callosotomy) due to the transport time and image acquisition time. The direct costs are increased due to the cost of the laser fibers, but the length of stay is shorter and patient comfort is improved (our unpublished data, 2018).

\section{Conclusions}

MRg-LITT is an alternative option to open surgery for disconnection of the corpus callosum for the treatment of drop attacks in patients with epilepsy. Outcomes may be comparable to those for open or endoscopic microsurgery, with minimal blood loss, decreased pain, and shorter hospital stay.

\section{References}

1. Andersen B, Rogvi-Hansen B, Kruse-Larsen C, Dam M: Corpus callosotomy: seizure and psychosocial outcome. A 39-month follow-up of 20 patients. Epilepsy Res 23:77-85, 1996

2. Beaumont TL, Mohammadi AM, Kim AH, Barnett GH, Leuthardt EC: Magnetic resonance imaging-guided laser interstitial thermal therapy for glioblastoma of the corpus callosum. Neurosurgery 83:556-565, 2018

3. Bogen JE, Fisher ED, Vogel PJ: Cerebral commissurotomy. A second case report. JAMA 194:1328-1329, 1965

4. Carpentier A, McNichols RJ, Stafford RJ, Guichard JP, Reizine D, Delaloge S, et al: Laser thermal therapy: real-time MRI-guided and computer-controlled procedures for metastatic brain tumors. Lasers Surg Med 43:943-950, 2011

5. Cendes F, Ragazzo PC, da Costa V, Martins LF: Corpus callosotomy in treatment of medically resistant epilepsy: preliminary results in a pediatric population. Epilepsia 34:910-917, 1993

6. Curry DJ, Gowda A, McNichols RJ, Wilfong AA: MRguided stereotactic laser ablation of epileptogenic foci in children. Epilepsy Behav 24:408-414, 2012

7. Diaz R, Ivan ME, Hanft S, Vanni S, Manzano G, Jagid J, et al: Laser interstitial thermal therapy: lighting the way to a new treatment option in neurosurgery. Neurosurgery 79 (Suppl 1):S3-S7, 2016

8. Eder HG, Feichtinger M, Pieper T, Kurschel S, Schroettner $\mathrm{O}$ : Gamma knife radiosurgery for callosotomy in children with drug-resistant epilepsy. Childs Nerv Syst 22:1012-1017, 2006

9. Esquenazi Y, Kalamangalam GP, Slater JD, Knowlton RC, Friedman E, Morris S-A, et al: Stereotactic laser ablation of epileptogenic periventricular nodular heterotopia. Epilepsy Res 108:547-554, 2014

10. Graham D, Tisdall MM, Gill D: Corpus callosotomy outcomes in pediatric patients: A systematic review. Epilepsia 57:1053-1068, 2016

11. Ho AL, Miller KJ, Cartmell S, Inoyama K, Fisher RS, Halpern $\mathrm{CH}$ : Stereotactic laser ablation of the splenium for intractable epilepsy. Epilepsy Behav Case Rep 5:23-26, 2016

12. Karsy M, Patel DM, Halvorson K, Mortimer V, Bollo RJ: Anterior two-thirds corpus callosotomy via stereotactic laser ablation. Neurosurg Focus 44 (VideoSuppl2):V2, 2018

13. Kawai K, Shimizu H, Yagishita A, Maehara T, Tamagawa $\mathrm{K}$ : Clinical outcomes after corpus callosotomy in patients with bihemispheric malformations of cortical development. J Neurosurg 101 (1 Suppl):7-15, 2004

14. Missios S, Bekelis K, Barnett GH: Renaissance of laser interstitial thermal ablation. Neurosurg Focus 38(3):E13, 2015

15. Pruitt R, Gamble A, Black K, Schulder M, Mehta AD: Com- 
plication avoidance in laser interstitial thermal therapy: lessons learned. J Neurosurg 126:1238-1245, 2017

16. Singh H, Essayed WI, Deb S, Hoffman C, Schwartz TH: Minimally invasive robotic laser corpus callosotomy: a proof of concept. Cureus 9:e1021, 2017

17. Sood S, Marupudi NI, Asano E, Haridas A, Ham SD: Endoscopic corpus callosotomy and hemispherotomy. J Neurosurg Pediatr 16:681-686, 2015

18. Tao JX, Issa NP, Wu S, Rose S, Collins J, Warnke PC: Interstitial stereotactic laser anterior corpus callosotomy: a report of 2 cases with operative technique and effectiveness. Neurosurgery [epub ahead of print], 2018

19. Torres-Reveron J, Tomasiewicz HC, Shetty A, Amankulor NM, Chiang VL: Stereotactic laser induced thermotherapy (LITT): a novel treatment for brain lesions regrowing after radiosurgery. J Neurooncol 113:495-503, 2013

20. Van Wagenen WP, Herren R: Surgical division of commissural pathways in the corpus callosum: Relation to spread of an epileptic attack. Arch Neurol Psychiatry 44:740-759, 1940

21. Wicks RT, Jermakowicz WJ, Jagid JR, Couture DE, Willie JT, Laxton AW, et al: Laser interstitial thermal therapy for mesial temporal lobe epilepsy. Neurosurgery 79 (Suppl 1):S83-S91, 2016

22. Wilson DH, Reeves A, Gazzaniga M: Division of the corpus callosum for uncontrollable epilepsy. Neurology 28:649653,1978

23. Wong TT, Kwan SY, Chang KP, Hsiu-Mei W, Yang TF, Chen
YS, et al: Corpus callosotomy in children. Childs Nerv Syst 22:999-1011, 2006

24. Xu DS, Chen T, Hlubek RJ, Bristol RE, Smith KA, Ponce FA, et al: Magnetic resonance imaging-guided laser interstitial thermal therapy for the treatment of hypothalamic hamartomas: a retrospective review. Neurosurgery 83:11831192,2018

\section{Disclosures}

The authors report no conflict of interest concerning the materials or methods used in this study or the findings specified in this paper.

\section{Author Contributions}

Conception and design: Palma, Couture. Acquisition of data: Palma. Analysis and interpretation of data: Palma. Drafting the article: Palma, Wicks, Couture. Critically revising the article: all authors. Reviewed submitted version of manuscript: all authors. Approved the final version of the manuscript on behalf of all authors: Palma. Study supervision: Couture.

\section{Correspondence}

Atilio E. Palma: Wake Forest University School of Medicine, Winston-Salem, NC. apalma@wakehealth.edu. 